\title{
Datos abiertos Open data
}

\author{
Edgar A. Ruvalcaba Gómez \\ Universidad de Guadalajara (México) \\ ORCID ID 0000-0003-0999-0680 \\ edgar.ruvalcaba@cucea.udg.mxnico.es
}
Cita recomendada:
Ruvalcaba Gómez, E. A. (2020). Datos abiertos. Eunomía. Revista en Cultura de la Legalidad, 18, pp. $327-$ 334.
doi: https://doi.org/10.20318/eunomia.2020.5280

Recibido / received: 03/06/2019

Aceptado / accepted: 19/11/2019

\begin{abstract}
Resumen
Los Datos Abiertos (DA) u Open Data están siendo un tema de alta relevancia en las administraciones públicas cuando se habla de innovación y modernización del sector público, no solo por su reciente disrupción, sino por su potencial en términos de valor público. La puesta en marcha de políticas que impulsan el acceso libre de datos está transformando, en gran medida, los servicios que ofrecen los gobiernos y generando un valor transformador sin precedentes. Este fenómeno se está dando principalmente bajo el nuevo modelo de gestión pública llamado Gobierno Abierto. En este sentido, este trabajo presenta una revisión sobre el concepto de DA principalmente vinculado a una idea de apertura gubernamental, así mismo se discute la relevancia de los DA y se hacen algunas reflexiones sobre el rol y las implicaciones políticoadministrativas que representan este tipo de datos.
\end{abstract}

\section{Palabras clave}

Datos abiertos, gobierno abierto, gestión pública, valor público.

\begin{abstract}
Open Data $(O D)$ is a topic of high relevance in public administrations related to innovation and modernization of the public sector, not only for its recent disruption, but for its potential in terms of public value. The implementation of policies that promote free data access is transforming the services offered by the government and generating unprecedented value. This phenomenon is occurring mainly under the new public management model called Open Government. In this sense, this paper presents a review of the concept of OD mainly linked to an idea of government openness, the relevance of $O D$ is also discussed and some reflections are made about the role and political-administrative implications that these data represent.
\end{abstract}

\section{Keywords}

Open data, open government, public management, public value. 
SUMARIO. 1. Introducción. 2. Marco teórico-conceptual. 3. Relevancia de los Datos Abiertos. 4. Discusión y reflexiones.

\section{Introducción}

Los modelos de gestión pública que ha asumido el aparato gubernamental han cambiado considerablemente debido fundamentalmente al uso social de las nuevas tecnologías, donde la información fluye en grandes cantidades y en tiempo real. En este sentido han surgido fenómenos disruptivos asociados a las tecnologías sociales que están revolucionado el sistema gubernamental, muestra de ello son los Datos Abiertos (DA) u Open Data. Los DA están cobrando relevancia bajo un enfoque más amplio conocido como «gobierno abierto», que es un modelo de gestión pública que busca estar en sintonía con una nueva realidad y, dar rumbo a un sector público que demanda mayor cooperación entre gobierno y sociedad.

En los últimos años, se ha presentado una tendencia en los gobiernos de diferentes niveles hacia liberar y publicar datos, no solo como un medio para generar transparencia y acceso a la información, sino también para impulsar el uso de la información en nuevas iniciativas que busquen mejorar la calidad de vida de los ciudadanos. Los principales beneficios de la apertura de los datos tienen que ver, por un lado, con la mejora en la calidad democrática, que tiene relación con tener acceso a datos que dé lugar a una participación ciudadana más informada. Por otro lado, encontramos el beneficio económico, el cual busca que iniciativas privadas agreguen valor a la información través de procesos de innovación que generen nuevos productos.

Cuando hablamos de DA debemos pensar en el contexto de apertura gubernamental como una nueva forma de entender la relación entre sociedad y gobierno, una idea donde la información pública y los datos son de libre acceso, sin restricción de derechos y con ciertas características que los hacen útiles para los ciudadanos. Los DA tienen las características de ser utilizados, reutilizados y redistribuidos libremente por cualquier persona para los fines que a ellos convengan. Esto nos habla de que la información debe estar disponible como un todo, normalmente descargándola de Internet de una forma accesible y en formatos manipulables.

La idea de asumir los DA gubernamentales como un tema de estudio ha crecido progresivamente desde el año 2008 cuando Murray-Rust (2008) comenzó a estudiarlo desde una perspectiva académica y práctica. Sin embargo, aumentó significativamente su relevancia cuando en el 2009 se inicia el lanzamiento de diversas plataformas de DA, abriendo una nueva ventana a la información gubernamental y agregando características de gran potencial y valor público.

En el caso de España los DA han encontrado un incremento drástico a partir de la entrada en vigor de la Ley 19/2013, de 9 de diciembre, de transparencia, acceso a la información pública y buen gobierno, que se publicó en el Boletín Oficial del Estado el 10 de diciembre de 2013. La Ley ha venido a garantizar el derecho de acceso a la información, así como a reconfigurar dependencias y procesos dentro de las entidades públicas como la Administración General del Estado, las Comunidades Autónomas, las Administraciones Locales, el Congreso, el Senado, el Tribunal Constitucional, agencias estatales, entidades públicas empresariales, entre otros organismos, que son sujetos de la ley. 
En el desarrollo de este trabajo se presenta un análisis de los DA desde diferentes miradas. En la siguiente sección se expone un marco teórico-conceptual en el que se debaten principalmente el concepto de DA y su emergencia dentro de un nuevo modelo de gestión pública como es el gobierno abierto. En la tercera sección se describen y analizan las razones por las cuales son relevantes los DA. Por último, en la cuarta sección, se establece una discusión en torno al rol que están jugando los DA hoy en día, principalmente enfocado a un modelo de gobierno dentro de las administraciones públicas, así como algunas reflexiones en torno al uso de los DA.

\section{Marco teórico-conceptual}

El tema de DA u Open Data ha emergido de marera disruptiva dentro de la era de la información. Los DA aparecen muy vinculados a la investigación en materia de gobierno abierto en años recientes y han supuesto un crecimiento drástico dentro de la literatura. En la actualidad los DA se han convertido en un pilar para materializar las estrategias de apertura gubernamental (Attard et al., 2015; Barry y Bannister, 2014; Conradie y Choenni, 2014; Janssen et al., 2012; Khayyat y Bannister, 2015; Linders, 2013; Luna-Reyes et al., 2014; Mellouli, 2014; Sayogo y Pardo, 2013; Safarov et al., 2017; Whitmore, 2014; Zuiderwijk et al., 2018). Este concepto ha sido sumamente asociado al gobierno abierto y ha logrado desvincularse conceptualmente de temas de transparencia, participación ciudadana y rendición de cuentas.

Con lo anteriormente señalado, se debe precisar que la apertura gubernamental va más allá de la transparencia como el simple hecho de revelar información, el cómo se revele esta información condiciona el potencial y la capacidad para hacer uso de la misma información (Ruijer et al., 2018). Es decir, no basta exclusivamente con la transparencia para lograr gobiernos abiertos, puesto que es indispensable que surjan incentivos de participación que promuevan la colaboración entre gobierno y sociedad civil organizada, y es ahí donde los DA están jugando un rol interactivo con la sociedad. En este sentido, es posible precisar que la transparencia y los DA no son lo mismo, pero tienen una relación intrínseca donde lo segundo potencializa a lo primero.

Los elementos básicos del gobierno abierto representan un debate permanente que depende de las perspectivas de los investigadores y los estudios que los respaldan. Diversos autores hablan del gobierno abierto como un tema en desarrollo y con poca investigación (Criado y Ruvalcaba-Gómez, 2016; Lee y Kwak, 2012: Oszlak y Kaufman, 2014). Estudios realizados en los últimos años han considerado que los DA tienen características diferentes e independientes para desvincularse de la transparencia (Abu-Shanab, 2015; Ruvalcaba-Gómez et al. 2018; Williamson, y Eisen, 2016).

Los DA configuran un gran recurso que está siendo cada vez más explotado por personas y organizaciones que, con diferentes tipos de datos gubernamentales, generan un uso práctico. Para definir el término de DA, Kassen (2013, p. 508), menciona que «The governmental open data project can be described as an official web-portal launched at the federal or local level aimed at making certain types of governmental datasets publicly accessible via Internet in a machine-readable format». Aquí encontramos una descripción simple y pragmática del tema para entender fácilmente la lógica de los DA dentro de los gobiernos. Por otro lado, se ha definido el open data como «un movimiento que promueve la liberación de datos, generalmente no textuales y en formatos reutilizables como csv (commaseparatedvalues), procedentes de organizaciones diversas» (Peset et al., 2011, p.165). 
Para el Open GovernmentWorkingGroup (2013) los principios de DA de gobierno son: 1. Completo. Todos los datos públicos están disponibles. Los datos públicos son datos que no están sujetos a restricciones de privacidad, seguridad o privilegios de algún tipo. 2. Primario. Los datos son recolectados de la fuente, con el nivel más alto posible de granularidad, no de manera agregada o de una forma modificada. 3. Oportuno. Los datos se ponen a disposición tan pronto como sea necesario para preservar su valor. 4. Accesible. Los datos están disponibles para una gama más amplia de usuarios y propósitos. 5. Procesables automáticamente. Los datos se estructuran razonablemente para permitir su procesamiento automático. 6 . No discriminación. Los datos están disponibles para cualquiera, sin requisitos de registro. 7 . No propietario. Los datos están disponibles en un formato sobre el cual ninguna entidad tiene un control exclusivo. 8. Licencia libre. Los datos no están sujetos a ningún derecho de autor, patentes, marcas o reglamento de secreto comercial. Se pueden permitir restricciones razonables de privacidad, seguridad y privilegio.

Partiendo de la premisa de que la información pública es de todos los ciudadanos y debe gozar de accesibilidad, se piensa en la forma de materializar dicha idea, aunque muchas veces los gobiernos no tienen capacidad para producir todo el valor social que los datos pueden ofrecer. Los ciudadanos o las empresas pueden hacer uso de esos datos para generar valor y servicios. De este modo los DA cumplen una función que va más allá de la simple transparencia o la rendición de cuentas, pues se convierten en herramientas asequibles y útiles a la sociedad.

Hoy en día las políticas de DA tienen el potencial de incentivar la participación y la interacción de los gobiernos con múltiples sectores y, también contribuyen a una integración social en donde los datos son ofrecidos en igualdad de condiciones para estimular el crecimiento económico y propiciar otros beneficios sociales (RuvalcabaGómez, 2017; Zhao y Fan, 2018; Zuiderwijk y Janssen, 2014). Existen iniciativas internacionales que han cobrado la atención de la comunidad global como Followthe Money, que consiste en una plataforma que rastrea todo el proceso del dinero público, permitiendo visualizar y manipular los datos que se producen en formato libre y abierto.

De forma sistemática surgen iniciativas de DA no solo a nivel nacional, sino también en niveles supranacionales como es el caso de la Unión Europea (https://data.europa.eu). Incluso a niveles subnacionales se puede encontrar una gran cantidad de casos como los de Vancouver (http://data.vancouver.ca/datacatalogue/cityStreets.htm), San Francisco (https://datasf.org) y New York (https://nycopendata.socrata.com), que destacan por ser pioneros en este ámbito. En España, hoy en día, se cuenta con múltiples iniciativas de datos abiertos a nivel regional y municipal. A nivel nacional destaca el sitio web https://datos.gob.es/, que configura un esfuerzo de diferentes dependencias gubernamentales y organizaciones de la sociedad civil.

\section{Relevancia de los Datos Abiertos}

El rol y la relevancia de los DA ha tenido un análisis teórico y empírico en los últimos años. Las iniciativas que se han puesto en marcha en este sentido han destacado su valor en diversos aspectos. En la lógica del modelo de gobierno abierto tiene como objetivo lograr un gobierno que permita la cooperación entre gobierno y sociedad, para que sea más transparente y democrático. Los DA no solo mejoran la transparencia y la rendición de cuentas de un gobierno, sino que también pueden generar beneficios económicos, soluciones innovadoras para el avance de la comunidad y el apoyo a las funciones de las administraciones públicas. Además, 
estos beneficios pueden lograrse simplemente publicando y reutilizando datos, que ya se han producido en el gobierno.

En base a lo anterior es posible asumir que las dos razones principales que motivan alos gobiernos a publicar DA son: a) el desarrollo de la calidad democrática $y, b)$ el desarrollo de la economía. Con respecto a la primera razón, los gobiernos explotan las iniciativas de DA para dar acceso a la información y ser más transparentes. La segunda razón, por otro lado, permite el crecimiento de la información y la generación de valor social y emprendedor. Si bien los datos confidenciales o personales no se pueden compartir por una protección jurídica, otros datos pueden tener un valor económico para empresas o individuos si se explotan. En este sentido, la publicación de datos, como datos de tráfico, meteorológicos, presupuestarios, geoespaciales y geográficos, brinda a los consumidores la oportunidad de crear nuevos servicios que, además de ser rentables, también pueden beneficiar el bien común.

Existen diferentes razones que dan sentido a la implementación de políticas de DA, estas razones tienen que ver con beneficios sociales, los cuales tienen implicaciones prácticas en la calidad de vida de una sociedad. Para el grupo de trabajo Open Government Data de la Open KnowledgeFoundation hay tres razones principales para abrir datos de carácter gubernamental:

1. Transparencia: para tener una sociedad democrática que funcione bien, los ciudadanos y otras partes interesadas deben poder monitorear las iniciativas gubernamentales y su legitimidad. La transparencia también significa que los interesados no solo pueden acceder a los datos, sino que también deben estar habilitados para usarlos, reutilizarlos y distribuirlos. El éxito para lograr la transparencia da como resultado un aumento considerable en el control social ciudadano.

2. Liberar valor social y comercial: los gobiernos son uno de los mayores productores y recolectores de datos en muchos aspectos. Todos los datos, ya sean direcciones de escuelas, datos geoespaciales, datos medioambientales, datos de transporte y planificación, o datos presupuestarios, tienen valor social y comercial. Estos pueden utilizarse para una serie de propósitos distintos que son diferentes de los previstos originalmente. Al publicar dichos datos, el gobierno alienta a las partes interesadas a innovar y crear nuevos servicios.

3. Gobernanza participativa: a través de la publicación de datos del gobierno, los ciudadanos tienen la oportunidad de participar activamente en los procesos de gobernanza, como la toma de decisiones y la adopción de políticas, en lugar de votar esporádicamente en una elección. A través de iniciativas de datos gubernamentales abiertos, como portales, las partes interesadas también pueden estar más informadas y ser capaces de tomar mejores decisiones.

En España a partir de la creación de la Ley de Transparencia en 2013, han surgido múltiples iniciativas que utilizan los DA como elemento fundamental para potenciar acciones gubernamentales. Como ejemplo destacado podemos mencionar el portal «Decide-Madrid», el cual liberó una serie de datos de forma estructurada, sin restricciones legales y sobre todo útiles para que los ciudadanos pueda tomar decisiones. Es importante mencionar que este tipo de iniciativas son impulsadas y demandadas por organizaciones de la sociedad civil como Fundación CIVIO y Transparencia Internacional. Incluso la misma Ley de Transparencia tuvo un trabajo coordinado con organizaciones para llegar a definir atributos de la información y datos, 
estableciendo la periodicidad, actualización, claridad, gratuidad, estructura, y calidad como características primordiales de los datos.

Los beneficios de los DA se traducen en una apertura a la información que implica mayor responsabilidad y que, a su vez, funciona como un dispositivo anticorrupción. Además, mediante la creación de nuevos servicios basados en DA, los usuarios agregan valor social, que también puede tener impacto e intereses comerciales. En este sentido, la participación de los ciudadanos en los procesos de toma de decisiones también es un aspecto muy importante de la apertura de datos gubernamentales, ya que permite que los gobiernos estén más centrados en las necesidades de los ciudadanos. Sin embargo, la participación ciudadana no solo se limita al proceso de toma de decisiones. Las iniciativas de gobierno abierto también pueden permitir a las partes interesadas proporcionar retroalimentación sobre las acciones del gobierno y, colaborar en la formulación de políticas.

\section{Discusión y reflexiones}

Es evidente la relevancia y trascendencia de los DA actualmente en los gobiernos y diversos sectores que trabajan con grandes cantidades de información. Las políticas de DA que impulsan muchas administraciones públicas experimentan una serie de obstáculos culturales que les impiden alcanzar su máximo potencial. En este sentido, existen gobiernos que no muestran la voluntad política de abrir los datos, en otros casos se carece del recurso humano capacitado para instrumentalizar estrategias de DA. Por otro lado, existen supuestos en los que hay una percepción de que la apertura de datos requiere una gran cantidad de recursos y esfuerzos, o que la publicación de datos del gobierno podría ser contraproducente. Sin embargo, estas ideas están cambiando paulatinamente en todo el mundo, principalmente debido a la presión de la sociedad civil y el isomorfismo institucional, que consiste en replicar prácticas de otras instituciones.

La idea positivista sobre los beneficios de los DA señala que al liberarse los datos se producirá crecimiento económico, mejoras en la democracia y mejores servicios públicos. La idea de «abrir por defecto» tiene sentido y ha cobrado gran peso dentro de la sociedad civil, que demanda más apertura democrática a los gobiernos (Carrara et al., 2015; Hossain et al., 2015). Sin embargo, existen algunas restricciones legales que limitan la apertura de datos debido a la protección de datos personales y a cuestiones de seguridad. Es importante analizar sobre el fenómeno de los DA, utilizando enfoques teóricos que permitan comprender los procesos de desarrollo e implementación, con el fin de obtener evidencias sobre la apertura y calidad de los datos bajo un enfoque de gobierno abierto.

Los DA muestran un potencial tan elevando que se ha llegado hablar de una revolución de datos (Melamed, 2014), esto implica un cambio disruptivo en la agenda de los gobiernos. Esta transformación podría tener efectos negativos si se llegara a salir de control, el poder de los datos cada día es mayor dentro de una era de la información, como la que estamos viviendo. En la actualidad, muchas sociedades son dependientes de sistemas tecnológicos que trabajan con datos, y aunque el tema de la protección de datos ha cobrado mucho auge en un sentido normativo, existen interés económicos y políticos que han sacado ventaja desleal de los datos. Considerando el peligro y fragilidad de manejar los datos con intereses perversos, es necesario y complicado valorar cuidadosamente la apertura de datos y el tipo de datos al que se busca tener acceso universal con el fin de generar beneficios sociales.

Analizando las tendencias en la gestión pública (Criado, 2016), es posible inferir que la adopción de una nueva perspectiva de gobernanza pública que impulsa 
los DA, está causando cambios en las políticas públicas de innovación, las cuales se están implementando bajo la concepción de un modelo de gobierno abierto. Aunque el impulso a la apertura de datos se encuentra en una fase emergente en cuanto a su implementación entre los gobiernos, su desarrollo ha sido disruptivo para los procesos de gestión pública. En los últimos años, España ha dado muestra de ser un país sumado en la tendencia de DA, y que de la misma manera sus gobiernos subnacionales están apostando por extraer beneficios de estas iniciativas.

Derivado de estas reflexiones es fundamental destacar la importancia de incorporar plataformas digitales, que establezcan bases de datos abiertas, estructuradas y organizadas para el uso de los ciudadanos. El gobierno abierto requiere una planificación estratégica de cara a lograr una traducción de la retórica y discursos políticos en acciones de política pública con valor social que promuevan la correcta explotación de los DA. Por otro lado, este fenómeno se puede entender desde la lógica del buen gobierno, de manera que la incorporación de iniciativas de DA también pueden considerarse como una búsqueda de legitimidad ante los problemas de opacidad y la percepción de corrupción de la ciudadanía.

Bibliografía

Attard, J., Orlandi, F., Scerri, S., y Auer, S. (2015). A systematic review of open government data initiatives. Government Information Quarterly, 32(4), 399-418.

Barry, E., y Bannister, F. (2014). Barriers to open data release: A view from the top. Information Polity: The International Journal of Government \& Democracy in the Information Age, 19(1), 129-152

Carrara, W., Chan, W. S., Fischer, S., y Steenbergen, E. van. (2015). Creating Value through Open Data: Study on the Impact of Re-use of Public Data Resources. European Commission.

Criado, J. I., y Ruvalcaba-Gómez, E. A. (2016). ¿Qué es y qué se entiende por Gobierno Abierto? Análisis de la percepción e implementación del Gobierno Abierto en el ámbito local español. Madrid, España: Laboratorio de Gobierno para la Innovación Pública.

Criado, J. I. (2016). Gobernanza inteligente, innovación abierta y tecnologías sociales en unas administraciones públicas colaborativas. ¿Hacia un cambio de paradigma en la gestión pública? En Nuevas tendencias en la gestión pública: Innovación abierta, gobernanza inteligente y tecnologías sociales en unas administraciones públicas colaborativas (pp. 22-48). Instituto Nacional de Administración Pública (INAP).

Conradie, P., y Choenni, S. (2014). On the barriers for local government releasing open data. Government Information Quarterly, 31, 1(0), S10-S17.

Hossain, M. A., Dwivedi, Y. K., y Rana, N. P. (2015). State of the Art in Open Data Research: Insights from Existing Literature and a Research Agenda. Journal of Organizational Computing and Electronic Commerce, 26(1), 14-40.

Janssen, M., Charalabidis, Y., y Zuiderwijk, A. (2012). Benefits, adoption barriers and myths of open data and open government. Information Systems Management, 29(4), 258-268.

Kassen, M. (2013). A promising phenomenon of open data: A case study of the Chicago open data project. Government Information Quarterly, 30(4), 508-513.

Khayyat, M., y Bannister, F. (2015). Open data licensing: More than meets the. Information Polity. The International Journal of Government \& Democracy in the Information Age, 20(4), 231-252.

Lee, G., y Kwak, Y. H. (2012). An open government maturity model for social mediabased public engagement. Government Information Quarterly, 29(4), 492-503. 
Linders, D. (2013). Towards open development: Leveraging open data to improve the planning and coordination of international aid. Government Information Quarterly, 30(4), 426-434.

Luna-Reyes, L. F., Bertot, J. C., y Mellouli, S. (2014). Open government, open data and digital government. Government Information Quarterly, 31(1), 4-5.

Melamed, C. (2014). A World That Counts Mobilisation the Data Revolution for Sustainable Development. New York. Retrieved from http://www.undatarevolution.org/wpcontent/uploads/2014/12/A-World-ThatCounts2.pdf

Mellouli, S., Luna-Reyes, L., y Zhang, J. (2014). Smart government, citizen participation and open data. Information Polity: The International Journal of Government \& Democracy in the Information Age, 19(1), 1-4.

Murray-Rust, P. (2008). Open data in science. Serials Review, 34(1), 52-64.

Open Government Working Group. (2013). World Bank's Open Government Data Working Group. "Open Data Toolkit". Published at data.worldbank.org/ogd

Oszlak, O. y Kaufman, E. (2014). Teoría y práctica del gobierno abierto. Red Gealc, OEA, IDRC. Organización de los Estados Americanos.

Peset, F., Ferrer-Sapena, A., y Subirats-Coll, I. (2011). Open data y linked open data: su impacto en el área de bibliotecas y documentación. El profesional de la información, 20(2),164-172.

Ruijer, E., Grimmelikhuijsen, S., van den Berg, J., y Meijer, A. (2018). Open data work: understanding open data usage from a practice lens. International Review of Administrative Sciences, 86(1) 3-19.

Ruvalcaba-Gómez, E. A. (2017). Perceptions about the Concept and Benefits of Open Government in Local Governments in Spain. Proceedings of the 10th International Conference on Theory and Practice of Electronic Governance (pp. 594-597). ACM.

Ruvalcaba-Gómez, E. A., Criado, J. I., y Gil-García, J. R. (2018). Discussing open government as a concept: a comparison between the perceptions of public managers and current academic debate. Proceedings of the 19th Annual International Conference on Digital Government Research: Governance in the Data Age (p. 71). ACM.

Safarov, I., Meijer, A., y Grimmelikhuijsen, S. (2017). Utilization of open government data: A systematic literature review of types, conditions, effects and users. Information Polity, 22(1), 1-24.

Sayogo, D. S., y Pardo, T. A. (2013). Exploring the determinants of scientific data sharing: Understanding the motivation to publish research data. Government Information Quarterly, 30, Supplement 1(0), S19-S31.

Whitmore, A. (2014). Using open government data to predict war: A case study of data and systems challenges. Government Information Quarterly, 31(4), 622-630.

Williamson, V., y Eisen N. (2016). The impact of open government: Assessing the evidence. Washington, DC, USA: The Brookings Institution.

Zhao, Y., y Fan, B. (2018). Exploring open government data capacity of government agency: Based on the resource-based theory. Government Information Quarterly, 35(1), 1-12.

Zuiderwijk, A., y Janssen, M. (2014). Open data policies, their implementation and impact: A framework for comparison. Government Information Quarterly, 31(1), 17-29.

Zuiderwijk, A., Janssen, M., y Davis, C. (2014). Innovation with open data: Essential elements of open data ecosystems. Information Polity: The International Journal of Government \& Democracy in the Information Age, 19(1), 17-33. 